\title{
Efecto de la hipertensión arterial en la función cognitiva de pacientes de 45 a 65 años. Hospital Luis Vernaza, Guayaquil, Ecuador
}

\author{
Impact of arterial hypertension on the cognitive function of patients between 45 and \\ 65 years. Luis Vernaza Hospital, Guayaquil, Ecuador
}

\author{
Gabriel A. Zúñiga-Salazar ${ }^{1}$, Sofía M. Hincapié-Arias' ${ }^{1}$ Erin E. Salazar-Bolaños ${ }^{1}$, Joffre J. Lara-Terán², \\ Silvia V. Cáceres-Vinueza ${ }^{3}$ y Yan C. Duarte-Vera ${ }^{3,4,5 *}$
}

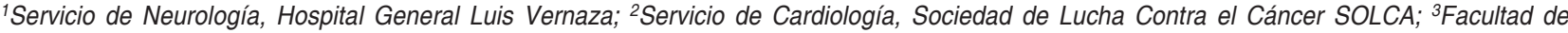
Ciencias Médicas, Universidad de Guayaquil; ${ }^{4}$ Servicio de Cardiología, Hospital General Luis Vernaza; ${ }^{5}$ Departamento de Investigación de la, Sociedad Ecuatoriana de Cardiología. Guayaquil, Ecuador

\begin{abstract}
Resumen
Introducción: El deterioro cognitivo leve es una alteración de la función cognitiva que no afecta de manera negativa a las actividades diarias de la persona. Factores de riesgo modificables como la hipertensión arterial podrían intervenir en el aceleramiento de este proceso y afectar a personas menores de 65 años. Una detección temprana del deterioro cognitivo leve por medio del Montreal Cognitive Assesment (MoCA) en personas con hipertensión arterial ayudaría a idear medidas para su control eficaz. Materiales y métodos: Se diseñó un estudio de tipo observacional transversal. La población se integra con pacientes hipertensos de 45 a 65 años de Guayaquil. De una base de datos de 570 pacientes, se realizó un llamado a sujetos que cumplieran los criterios de inclusión y exclusión, para la evaluación de la función cognitiva por medio del MoCA. Resultados: El 93.3\% de los participantes sometidos a la prueba MoCA presentó un puntaje menor de 26. El promedio del puntaje total fue de 18.9 y el $51.6 \%$ de los participantes reveló deterioro cognitivo leve. Se obtuvo una correlación negativa del $40 \%(-0.40)$ con una $p=0.0015$ entre años con hipertensión arterial y el resultado total de la prueba MoCA. Conclusiones: Por medio del MoCA se pudo cuantificar la magnitud del deterioro cognitivo en estos pacientes. Se identificó que la mayoría de los participantes examinados presentaba un puntaje promedio menor del intervalo normal. Las cifras obtenidas de los valores de correlación de Pearson muestran una actividad tendencial y relación de la hipertensión arterial con el deterioro de la función cognitiva.
\end{abstract}

Palabras clave: Deterioro cognitivo leve. Hipertensión arterial. MoCA. Demencia. Memoria. Función cognitiva.

\begin{abstract}
Introduction: Mild cognitive impairment is an alteration of cognitive function that does not negatively affect the daily activities of the person. Modifiable risk factors such as hypertension could be involved in the acceleration of this process affecting
\end{abstract}

Disponible en internet: 10-02-2020 Arch Cardiol Mex. 2020;90(3):284-292 www.archivoscardiologia.com BY-NC-ND (http://creativecommons.org/licenses/by-nc-nd/4.0/). 
people under 65 years of age. Early detection of mild cognitive impairment through the Montreal Cognitive Assessment (MoCA) in people with high blood pressure, would help to formulate strategies for its effective control. Materials and methods: A cross-sectional observational study was designed. The population is hypertensive patients between 45 and 65 years old in Guayaquil. From a database of 570 patients, a call was made to patients who met the inclusion and exclusion criteria, for the evaluation of cognitive function through MoCA. Results: $93.3 \%$ of the participants who underwent the MoCA test had a score lower than 26. The average of the total score was 18.9 , with $51.6 \%$ of the participants suffering from mild cognitive impairment. A negative correlation of $40 \%(-0.40)$ was obtained with a $p=0.0015$ between years with arterial hypertension and the total result of the MoCA test. Conclusions: Through MoCA, the magnitude of cognitive deterioration in these patients could be quantified. It was detected that the majority of the examined participants had a lower average score of the normal range. The figures obtained from the Pearson correlation values show trend activity, relating arterial hypertension and deterioration of cognitive function.

Key words: Mild cognitive impairment. Arterial hypertension. MoCA. Dementia. Memory. Cognitive function.

\section{Introducción}

Las tasas de mortalidad entre los individuos mayores han disminuido, mientras que la expectativa de vida ha aumentado; en consecuencia, se han incrementado los casos de demencia en el plano global. La demencia es un síndrome caracterizado por la pérdida progresiva e irreversible de las funciones cognitivas: atención, memoria, función ejecutiva, habilidad visual-espacial y lenguaje, e interfiere con el estilo de vida de las personas que la padecen y altera a aquellas que pertenecen a su círculo familiar, lo que la convierte en una amenaza mayor de salud pública1. Ésta se presenta por lo regular en sujetos mayores de 65 años. En el 2015 existían 46.8 millones de personas con demencia en el mundo. En las Américas había en el 2015 un total de 9.4 millones de individuos con demencia ${ }^{2}$. En el 2010 en Ecuador, el $20.3 \%$ de los sujetos de 60 años y mayores mostró deterioro cognitivo ${ }^{3}$.

Antes de llegar a la demencia, los pacientes pasan por un estado intermedio, entre la cognición normal y la demencia, llamado deterioro cognitivo leve, que no interfiere con las actividades diarias normales de las personas ${ }^{4}$. Éste es más común a medida que se envejece, pero factores de riesgo modificables como la hipertensión arterial pueden intervenir en el aceleramiento de este proceso y afectar a individuos menores de 65 años. Numerosos estudios han demostrado la relación entre la hipertensión arterial y la demencia, pero todavía es escasa la investigación acerca de la existencia de deterioro cognitivo leve en pacientes hipertensos menores de 65 años ${ }^{5}$.

Una identificación temprana del deterioro cognitivo leve por medio de pruebas de detección como el MoCA (Montreal Cognitive Assesment) en pacientes con hipertensión arterial ayudaría a conformar tratamientos para su control eficaz y lentificar el proceso del deterioro cognitivo, además de establecer esquemas que ayuden a reducir el efecto de la posible demencia en estos pacientes, debido a que se ha observado que la modificación intensiva de los factores de riesgo, en especial entre los 45 y 65 años de edad, tiene el potencial de retrasar o prevenir un número considerable de casos de demencia en el plano mundial ${ }^{6,7}$.

\section{Deterioro cognitivo leve}

El término de deterioro cognitivo leve (DCL) se refiere a la alteración de la función cognitiva que no cumple los criterios de demencia. En la demencia no sólo hay alteración de la función cognitiva, sino también afectación negativa de las actividades diarias del individuo, mientras que en el deterioro cognitivo no existe alteración de la vida diaria. Este tipo de deterioro puede clasificarse de varias maneras; la de mayor utilidad cataloga el trastorno en los tipos amnésico y no amnésico ${ }^{8}$.

\section{Tipos de deterioro cognitivo leve}

El DCL se divide en los tipos amnésico y no amnésico y cada uno se subdivide en dominio único y dominios múltiples. El DCL de tipo amnésico es el más común y se relaciona más con enfermedad de Alzheimer. Los criterios que de manera original se idearon para el deterioro cognitivo leve se usan en la actualidad para definir el tipo amnésico de un dominio único ${ }^{9}$. Entre estos criterios figuran los siguientes:

- Queja de memoria, preferiblemente corroborada por un informante

- Disminución de la memoria objetiva (para la edad y la educación) 
- Función cognitiva general conservada

- Actividades intactas de la vida diaria

- Ausencia de demencia

En el caso del tipo amnésico de dominios múltiples existen alteraciones de otros dominios cognitivos que se revelan luego de un estudio neuropsicológico realizado de manera adecuada. Este tipo de DCL ocurre en los individuos que pueden padecer una leve alteración de sus actividades diarias, pero sin incurrir en los criterios para considerarlos dementes ${ }^{10}$.

Por otro lado, el DCL no amnésico, al igual que el anterior, se subclasifica en un subtipo único y un subtipo que analiza varios dominios. El primero se caracteriza por alteración en un dominio no relacionado con la memoria, es decir, se relaciona con la función ejecutiva, lenguaje o capacidad visual-espacial. Los pacientes de este grupo parecen tener menor riesgo de conversión a la demencia. Según sea el dominio, las personas con este subtipo de DCL pueden progresar a otros síndromes, como la demencia frontotemporal, la afasia progresiva primaria, la demencia con cuerpos de Lewy, la parálisis supranuclear progresiva o la degeneración corticobasal ${ }^{11}$.

En el caso del DCL de tipo no amnésico de múltiples dominios, los pacientes que satisfacen estos criterios se ven afectados en varios dominios, con una relativa preservación de los problemas de memoria. Al parecer, en estos casos hay una vinculación con trastornos degenerativos como la demencia con cuerpos de Lewy o la demencia frontotemporal, que se relacionan con las proteínas $\tau$, TDP-43 y sinucleína $\alpha^{12}$.

Existe gran variabilidad acerca de la epidemiología del deterioro cognitivo leve, debido a los diferentes tipos de pruebas utilizados para valorarla, la pérdida de participantes y la aleatoridad del estudio. Por otro lado, los valores difieren mucho entre sí debido a las poblaciones conformadas, en virtud de su mayor prevalencia en edades mayores. En este caso existe mucha diferencia al comparar los resultados de pacientes de 40 a 60 años con sujetos de 60 a 70 . El intervalo aproximado es de 5 a 13 personas por cada 1,000 que sufren deterioro cognitivo leve cada año.

En Ecuador existen pocos estudios acerca de la incidencia o prevalencia del deterioro cognitivo leve, sobre todo en poblaciones menores de 65 años. En un análisis hospitalario realizado en el 2012 se observó que, de 212 pacientes, el $15 \%$ mostraba deterioro cognitivo leve en una población de 65 a 85 años. Entre los factores de riesgo vinculados con esta alteración se encuentran hipertensión, depresión, grado de educación y edad más avanzada.
En relación con la función cognitiva, la principal queja de los pacientes con DCL son los problemas de memoria, como recordar nombres, lugares o acciones realizadas minutos antes. A diferencia de los pacientes con demencia o enfermedad de Alzheimer, los enfermos con DCL se encuentran por lo general preocupados por sus alteraciones nemónicas.

En cuanto a los síntomas neuropsiquiátricos, se identifican manifestaciones clínicas relacionadas con pacientes con DCL, como irritabilidad, ansiedad, apatía, agresividad (la depresión es la más común de todas). Estos síntomas se presentan de modo leve y la forma de presentación es lo que diferencia la demencia de la enfermedad de Alzheimer, en la cual los síntomas se observan con mayor gravedad y frecuencia ${ }^{13}$.

En cuanto a la frecuencia con que aparece la depresión en pacientes con DCL, algunos estudios han observado que la depresión y su intensidad son mayores en los individuos con DCL y una atenuación progresiva de su función cognitiva ${ }^{14}$.

El diagnóstico de DCL se puede realizar mediante neuroimágenes o pruebas de laboratorio. La neuroimagen regular no se recomienda en la evaluación clínica habitual de DCL. Se puede solicitar como parte de una investigación para determinar las causas y el pronóstico del deterioro cognitivo leve. Entre los posibles estudios, la resonancia magnética estructural puede ser útil para identificar DCL y en los sujetos con mayor probabilidad de progresar del DCL a la demencia. Una de las imágenes que puede visualizarse en una resonancia magnética y que indica DCL es la medida de volumen del hipocampo y en ella se observa atrofia, que además se relaciona con riesgo de progresión a demencia. En general, la resonancia magnética no se utiliza como imagen sistemática para determinar DCL, pero según el historial clínico puede emplearse como herramienta para descartar anomalías como hematomas subdurales, episodios cerebrovasculares, neoplasias cerebrales y otras causas más. Otro tipo de neuroimagen útil para investigar el DCL es la tomografía por emisión de positrones con fluorodesoxiglucosa. Este tipo de imagen tiene utilidad en pacientes con DCL causado por enfermedad de Alzheimer, debido a que se detectan regiones de hipometabolismo en el cerebro y, en casos más avanzados, presencia de amiloide $^{15}$.

En cuanto a los estudios de laboratorio, se efectúan análisis de sangre generales, como biometría hemática, electrólitos, glucemia, calcio, hormonas tiroideas, vitamina $B_{12}$ y folato para descartar otras alteraciones 
y reconocer las causas más comunes del deterioro cognitivo. Según Clarfield, se observó en un metaanálisis que alrededor del $9 \%$ de las causas de deterioro cognitivo se debe a factores reversibles, entre ellos infecciones, hipermagnesemia, hiperglucemia, deficiencia de vitamina $B_{12} 0$ alteraciones de la función hepática. Otro estudio de laboratorio es el análisis del líquido cefalorraquídeo. Esta prueba es de utilidad para detectar la proteína $\tau$ en pacientes con altas probabilidades de demencia futura ${ }^{16}$.

En la actualidad no hay tratamiento farmacológico aprobado para tratar el DCL; sólo se controlan los factores de riesgo de los pacientes, se proporciona información y se emiten recomendaciones para maximizar el bienestar al reducir dichos factores. Los síntomas de depresión se atenúan con ayuda del tratamiento antidepresivo. Aunque es necesario tratar los síntomas depresivos, se ha documentado un efecto negativo en la función cognitiva, sobre todo en el adulto mayor, con la prescripción de anticolinérgicos ${ }^{17}$. Algunos estudios evalúan la eficacia de ciertos medicamentos para evitar la progresión del DCL; entre ellos figuran el uso de inhibidores de acetilcolinesterasa en pacientes con alto riesgo de padecer enfermedad de Alzheimer en el futuro. Por ahora no hay estudios que aprueben su consumo en los pacientes ${ }^{18}$.

\section{Materiales y métodos}

\section{Hipótesis de la investigación}

La hipertensión arterial se relaciona con un deterioro temprano de la función cognitiva en personas de mediana edad.

\section{Objetivo general}

- Determinar el efecto de la hipertensión arterial en la función cognitiva de pacientes de 45 a 65 años.

\section{Objetivos específicos}

- Detectar el deterioro de la función cognitiva en sujetos hipertensos de 45 a 65 años.

- Cuantificar la magnitud del deterioro de la función cognitiva en pacientes hipertensos de 45 a 65 años.

- Especificar los elementos de la función cognitiva que más se afectan en hipertensos de 45 a 65 años.

- Establecer un nexo entre el tiempo con hipertensión arterial y el deterioro cognitivo.

\section{Participantes}

De una base de datos de 570 pacientes, obtenida del Servicio de Cardiología de la consulta externa del Hospital Luis Vernaza, 145 individuos satisfacían los criterios de inclusión y exclusión, de los cuales sólo 60 completaron las pruebas y aceptaron ser parte del estudio realizado de acuerdo con las líneas directivas de la buena práctica clínica y las normas éticas para la experimentación humana establecidas por la Declaración de Helsinki. Cada paciente concedió su consentimiento informado para su inclusión en este estudio. El resto de las variables se obtuvo de la base de datos incluida en el sistema informático del hospital o a partir de la historia clínica del paciente (Fig. 1).

\section{Criterios de inclusión}

Hombres y mujeres de mediana edad (45 a 65 años) con diagnóstico previo de hipertensión arterial e individuos con un grado de educación mínimo de primaria.

\section{Criterios de exclusión}

Individuos con antecedentes de ictus, demencia, algún grado de discapacidad que impidiera realiza la prueba MoCA, enfermedad renal crónica, tratamiento actual con hemodiálisis, antecedentes de traumatismos craneoencefálicos tratados en unidad de cuidados intensivos, alcoholismo y depresión.

\section{Medición de la presión arterial}

La presión arterial se cuantificó con un esfigmomanómetro digital (OMRON HEM7130) antes de la evaluación de los pacientes con el MoCA. Los individuos se sentían cómodos y permanecieron sentados durante al menos cinco minutos antes de la medición. Ésta se llevó a cabo con el individuo sentado.

\section{Prueba neurocognitiva}

La prueba neurocognitiva de elección en el estudio fue el Montreal Cognitive Assesment (MoCA). El evaluador, que en este caso fue uno de los investigadores, realizó la prueba de forma directa y se efectuó en uno de los consultorios del área de cardiología en la consulta externa del hospital de manera individual y $\sin$ distracciones. El evaluador obtuvo e interpretó los resultados según las instrucciones de la prueba. 


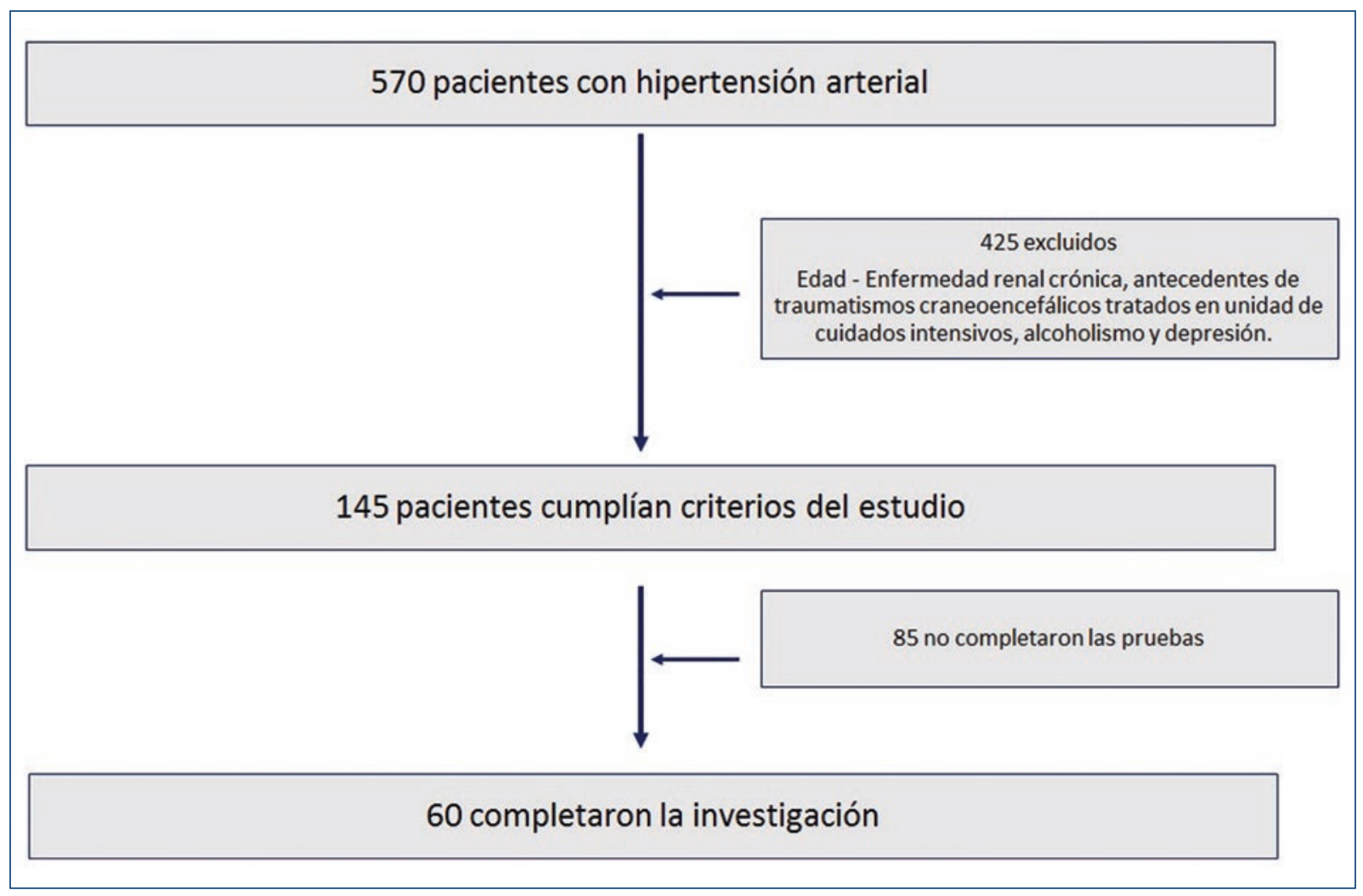

Figura 1. Diagrama de flujo para pacientes con hipertensión arterial.

La duración máxima de la prueba según el instructivo era no mayor de 10 minutos. El máximo puntaje es de 30 puntos. Un puntaje igual o mayor de 26 se consideró normal; un puntaje de 18 a 25 representó deterioro cognitivo leve; uno de 10 a 17 significó deterioro cognitivo moderado; y uno menor de 10 se asumió como deterioro cognitivo grave.

El MoCA evalúa las siguientes funciones cognitivas: visuoespacial/ejecutiva, identificación, memoria, atención, lenguaje, abstracción, recuerdo diferido y orientación.

\section{Diseño y estadística}

Se diseñó un estudio de tipo observacional transversal. La población en este caso se integró con los pacientes hipertensos de 45 a 65 años atendidos en la consulta externa del Hospital Luis Vernaza de Guayaquil en el periodo de 2017 a 2018 (tabla 1). Por medio de una base de datos recopilada y realizada en Excel se ejecutaron los análisis con el programa estadístico RStudio y se graficaron los resultados en STATA.

\section{Resultados}

De los pacientes sometidos a la prueba MOCA, $63 \%$ (38) correspondió a mujeres. El intervalo de edad de este estudio fue de 45 a 65 años, con 58 años la edad como promedio de los examinados. Al sumar todos los puntos en la prueba MOCA se obtiene un máximo de 30 puntos y es normal un puntaje mayor o igual a 26 . Se añade un punto si el examinado tiene 12 años o menos de estudios. Tal y como se observa en la figura 2, el $93.3 \%$ de los participantes tuvo un puntaje menor de 26.

Se registró un promedio de los puntajes obtenidos no sólo en la totalidad del estudio, sino de cada una de las áreas que compone la prueba MOCA. El promedio del puntaje total fue de 18.9, con una mediana de 18.5 y una desviación estándar de 4.35 (Fig. 3)

Se pueden distinguir tres grados de profundidad del deterioro cognitivo según la prueba MoCA: leve, moderado y grave (tabla 2). En la tabla 3 se detalla el porcentaje de la puntuación de cada una de las funciones cognitivas: en el área visoespacial/ejecutiva, el puntaje promedio fue de 2.36 , con un $47.2 \%$ de la nota 
Tabla 1. Características demográficas de la muestra

\begin{tabular}{|l|c|}
\hline Variable & Pacientes evaluados $\mathbf{n}=\mathbf{6 0}$ \\
\hline Edad, media & 58 \\
\hline Mujeres, $\mathrm{n}(\%)$ & $38(63)$ \\
\hline PAS, media en mmHg & 136.28 \\
\hline PAD, media en mmHg & 80.9 \\
\hline PAM, media en mmHg & 99.36 \\
\hline Años de HTA, media (años) & 8.2 \\
\hline Diabetes mellitus, n (\%) & $10(16.6)$ \\
\hline Dislipidemia, $\mathrm{n}(\%)$ & $14(23.3)$ \\
\hline Hipotiroidismo, $\mathrm{n}(\%)$ & $8(13.3)$ \\
\hline Tabaquismo, $\mathrm{n}(\%)$ & $4(6,6)$ \\
\hline Educación primaria, $\mathrm{n}(\%)$ & $18(30)$ \\
\hline Educación secundaria, $\mathrm{n}(\%)$ & $26(43)$ \\
\hline Educación superior, $\mathrm{n}(\%)$ & $16(27)$ \\
\hline
\end{tabular}

PAS: presión arterial sistólica; PAD: presión arterial diastólica; PAM: presión arterial media; HTA: hipertensión arterial.

total de esa área. En el área denominación se obtuvo un promedio de 2.23 y un $74.3 \%$ de la nota total del área. El área de memoria no incluye puntos. El área de atención se divide en tres partes, con un promedio total de 3.56 y $59.3 \%$ del puntaje. El área de lenguaje se divide en dos partes, con un promedio total de 1.46 de puntaje y un $48.6 \%$ del puntaje de esa área. En el área de abstracción se registró un promedio de puntos de 1.25 y un $62.5 \%$ del puntaje. En el área de recuerdo diferido se identificó un promedio de 1.8 puntos y un porcentaje de $36 \%$ del puntaje. En el área de orientación se identificó un promedio de 5.65 puntos y $94.1 \%$ del puntaje. El resultado más negativo en las pruebas fue el del grupo femenino con un puntaje promedio de 18.55. En los hombres, el puntaje promedio fue de 19.5 .

En cuanto al grado de educación, 18 participantes tuvieron una educación menor de 13 años, 26 estudiaron hasta la secundaria y 16 tenían título de educación superior. El grado de educación primaria se acompaña de puntajes más bajos en la prueba con un promedio de 16.88 y le sigue la educación secundaria con 19.57 y la superior con 20.06 (tabla 4). Se efectuó un análisis de correlación mediante el parámetro de Spearman entre los años de educación y el resultado total de la prueba MoCA. Existe una correlación positiva del 31\% (0.31) con una $p=0.01$. Esto significa que cuantos más

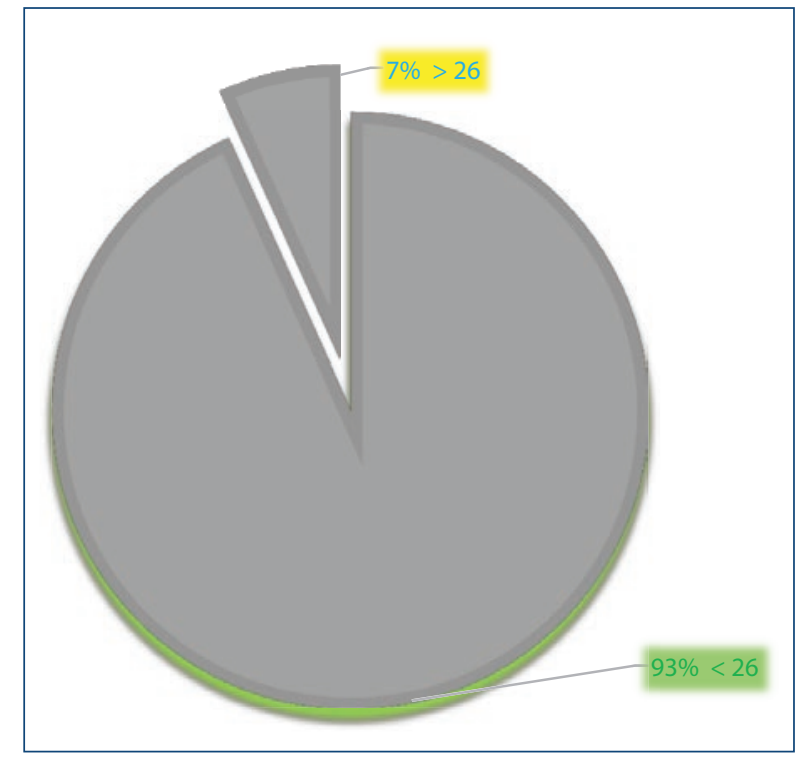

Figura 2. Representación del porcentaje de pacientes que tuvieron un puntaje mayor o menor de 26 en la prueba MoCA.

años de educación tenga la persona, mayor es el puntaje. Aunque la correlación no es alta, podría justificar una mayor investigación (Fig. 4).

Todos los participantes del estudio tenían hipertensión arterial. El promedio de la presión arterial media antes de realizar el examen fue de $99.36 \mathrm{mmHg}$, el de la presión arterial sistólica de $136.28 \mathrm{mmHg}$ y el de la presión arterial diastólica de $80.9 \mathrm{mmHg}$. Se correlacionó mediante el instrumento de Pearson la presión arterial sistólica al momento del examen y el resultado final de la prueba MoCA. Se obtuvo una correlación negativa del $26 \%(-0.26)$ con una $p=0.038$; es decir, a mayor presión arterial sistólica, menor puntaje total. Aunque la correlación no es alta, podría indicar una mayor investigación a futuro. La presión arterial diastólica no se correlaciona con un puntaje menor (Fig. 5).

El promedio de duración en años de la hipertensión arterial desde el momento de su diagnóstico fue de 8.2 años. Se correlacionaron mediante el parámetro de Spearman los años con hipertensión arterial y el resultado total de la prueba MoCA. Existe una correlación negativa del $40 \%(-0.40)$ con una $p=0.001$; esto significa que mientras más años haya hipertensión arterial, menor es el puntaje en la prueba MoCA. Aunque la correlación no es alta, podría justificar una mayor investigación (Fig. 6).

El $16.6 \%$ (10) de los participantes posee diabetes mellitus tipo II además de hipertensión arterial. El 23.3\% 


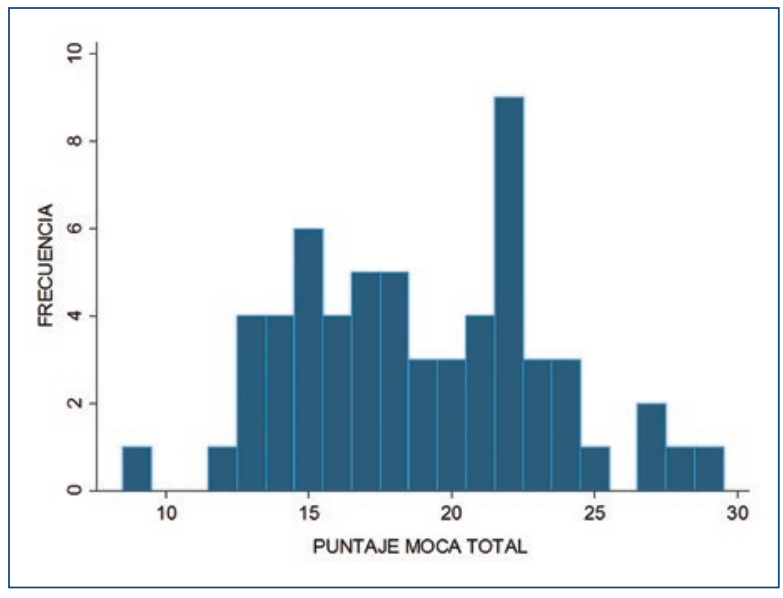

Figura 3. Histograma que representa el puntaje total de la prueba MoCA y su frecuencia.

Tabla 2. Gravedad del deterioro cognitivo según la prueba MoCA

\begin{tabular}{|l|c|c|c|}
\hline $\begin{array}{l}\text { Grados de } \\
\text { deterioro cognitivo }\end{array}$ & $\begin{array}{c}\text { Puntaje } \\
\text { MoCA }\end{array}$ & $\begin{array}{c}\text { Frecuencia } \\
\text { de pacientes }\end{array}$ & $\begin{array}{c}\text { Porcentaje } \\
\text { de pacientes }\end{array}$ \\
\hline Leve & $18-25$ & 31 & $51.6 \%$ \\
\hline Moderado & $10-17$ & 24 & $40 \%$ \\
\hline Grave & $<10$ & 1 & $1.6 \%$ \\
\hline
\end{tabular}

Tabla 3. Porcentaje de la puntuación de cada una de las funciones cognitivas

\begin{tabular}{|l|c|c|}
\hline & Promedio & Porcentaje \\
\hline Puntaje ejecutiva & 2.37 & $47.20 \%$ \\
\hline Puntaje identificación & 2.23 & $74.33 \%$ \\
\hline Puntaje atención & 3.57 & $59.33 \%$ \\
\hline Puntaje lenguaje & 1.47 & $48.66 \%$ \\
\hline Puntaje abstracción & 1.25 & $62.50 \%$ \\
\hline Puntaje recuerdo diferido & 1.8 & $36 \%$ \\
\hline Puntaje orientación & 5.65 & $94.16 \%$ \\
\hline
\end{tabular}

(14) tiene como comorbilidad dislipidemia, en tanto que $13.3 \%$ (8) padece hipotiroidismo. Otras anomalías de importancia no se mencionan, dado que constan en los criterios de exclusión establecidos para el estudio (tabla 1).

\section{Discusión}

Los resultados muestran una relación entre la hipertensión arterial y el deterioro cognitivo en este grupo poblacional, lo que confirma la hipótesis del estudio.

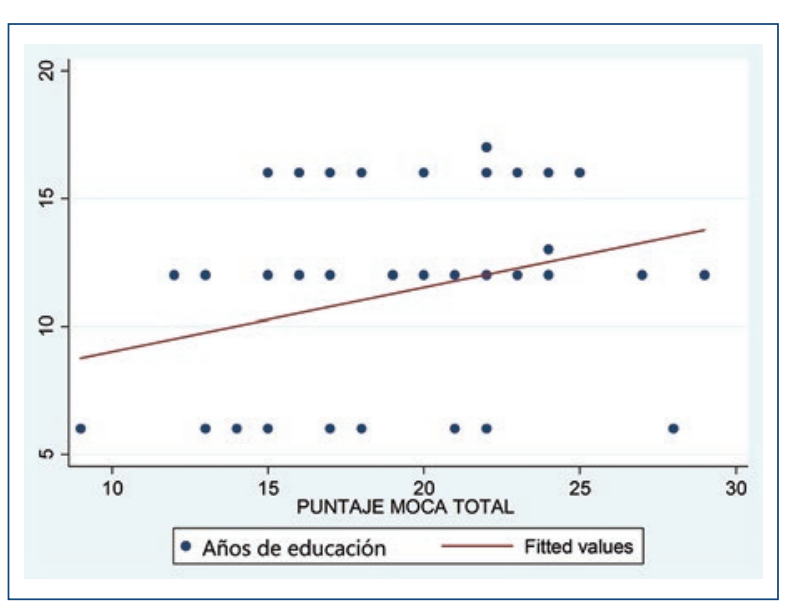

Figura 4. Diagrama de dispersión que representa la correlación entre años de educación y puntaje total de la prueba MoCA.

Tabla 4. Grado de educación y promedio del puntaje MoCA

\begin{tabular}{|l|c|c|}
\hline Grado de educación & $\begin{array}{c}\text { Frecuencia de } \\
\text { pacientes }\end{array}$ & $\begin{array}{c}\text { Promedio del } \\
\text { puntaje MoCA }\end{array}$ \\
\hline Primaria & 18 & 16.88 \\
\hline Secundaria & 26 & 19.57 \\
\hline Superior & 16 & 20.06 \\
\hline
\end{tabular}

Así como este protocolo, otros como los de Guo, et al., e In't Veld, et al. han demostrado con anterioridad un nexo e incluso un factor causal de la hipertensión con el deterioro cognitivo ${ }^{19}$. Las investigaciones previas se centran en adultos mayores (> 65 años), a diferencia de esta investigación que apunta hacia una población menor (45 a 65 años) y menos estudiada ${ }^{20}$.

Tzourio, et al. y Gupta et al. estudiaron el deterioro cognitivo en personas hipertensas de mediana edad y registraron un deterioro en $8.3 \%$ y $14 \%$, respectivamente. El porcentaje bajo, en comparación con el de este estudio, quizás podría estar relacionado con la utilización del Mini Mental State Examination que es menos sensible para detectar deterioro cognitivo leve ${ }^{21}$. En un estudio de Muela, et al., la prueba MoCa fue más sensible que el MMSE para identificar deterioro cognitivo leve en pacientes hipertensos ${ }^{22}$. En este estudio, en orden de mayor a menor afectación, las áreas más alteradas fueron: memoria a través del recuerdo diferido, ejecutiva, lenguaje, atención, abstracción, identificación y orientación.

Vicario, et al. ${ }^{23}$ mostraron un efecto de la hipertensión en la función ejecutiva frente a pacientes 


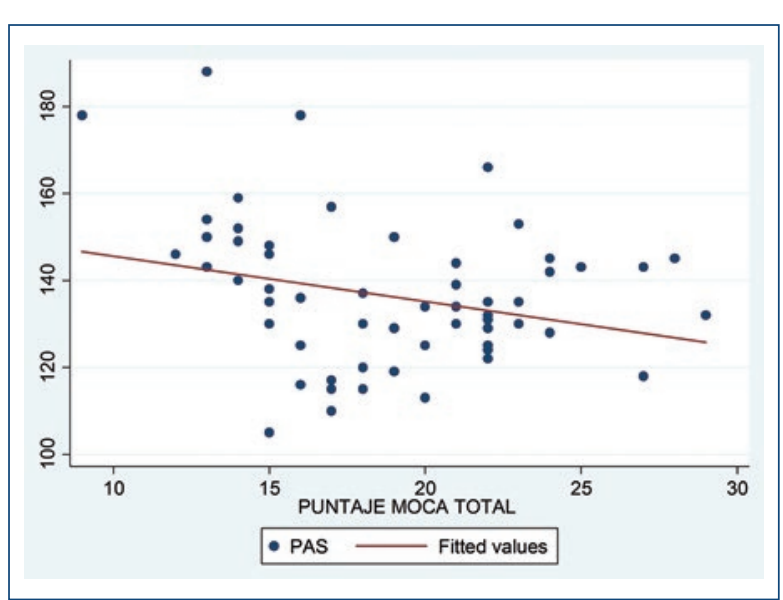

Figura 5. Diagrama de dispersión que muestra la correlación entre la PAS y el puntaje total de la prueba MoCA.

normotensos. Yaffe, et al., Swan, et al. e ladecola, et al. demuestran una evidencia moderadamente sólida para sustentar la afirmación de que la velocidad de procesamiento y la función ejecutiva disminuidas son los cambios cognitivos más comunes relacionados con la hipertensión ${ }^{24}$.

Los participantes de este estudio, además de hipertensión arterial, presentaban otros factores de riesgo que pueden influir en la función cognitiva. Livingston, et al. señalan en su estudio del 2017 los principales factores de riesgo que, al combinarse, pueden llevar a un deterioro de la función cognitiva. Éstos son bajo grado educativo, hipertensión de mediana edad, obesidad de mediana edad, pérdida de audición, depresión de la vida tardía, diabetes, inactividad física, tabaquismo y aislamiento social. En este estudio, los factores de riesgo presentes fueron antecedentes de tabaquismo, diabetes mellitus tipo II, dislipidemia, hipotiroidismo y bajo grado educativo; el mayor control fue la hipertensión arterial por su mayor tiempo de diagnóstico y tratamiento ${ }^{25}$.

Debido a una imposición estricta de criterios de inclusión y exclusión, y a la disposición de los participantes a realizar el estudio, se obtuvo una población de 60. A pesar de este bajo número de población, las cifras obtenidas de los valores de correlación de Pearson y Spearman muestran una actividad tendencial que relaciona la hipertensión arterial y el deterioro de la función cognitiva, cuya tendencia se confirmaría con estudios similares de mayor población.

Las limitaciones de este protocolo corresponden a la naturaleza de la prueba utilizada, ya que no establece

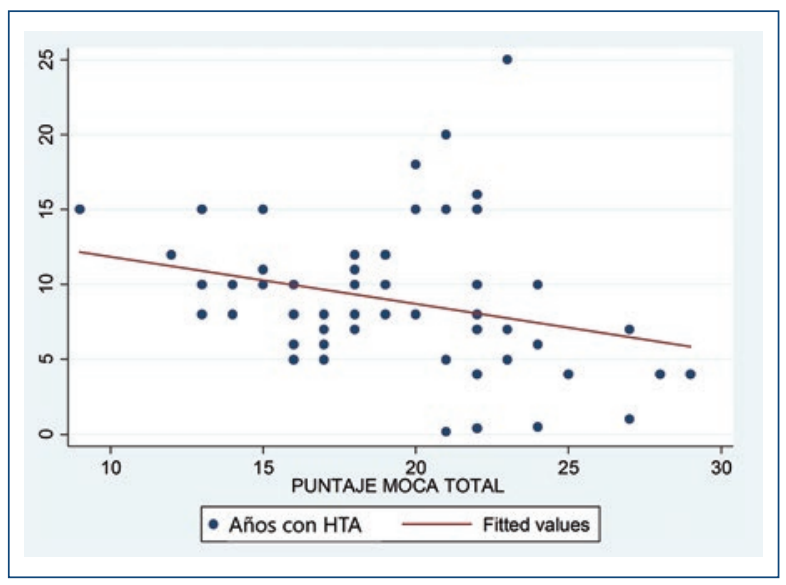

Figura 6. Diagrama de dispersión que representa la correlación entre años con HTA y el puntaje total de la prueba MoCA.

un diagnóstico definitivo, sino una detección de deterioro cognitivo leve. Debido a que es un estudio de detección de DCL, se limita a predecir con certeza el pronóstico de los participantes, por lo que se requiere seguimiento de éstos para delinear su desarrollo en relación con su función cognitiva.

\section{Conclusiones}

En este estudio se observó el efecto que ocasiona la hipertensión arterial en la función cognitiva de pacientes de 45 a 65 años. Se detectó que la mayoría de los participantes analizados, que cumplían con los criterios de inclusión y exclusión, presentaban un puntaje promedio menor del intervalo normal. Por medio de la prueba MoCA se pudo cuantificar la magnitud del deterioro cognitivo en estos pacientes y, junto con las correlaciones de Spearman y Pearson, observar la actividad tendencial en relación con la hipertensión arterial y la función cognitiva.

\section{Agradecimientos}

Los autores expresan su agradecimiento a la Sociedad Ecuatoriana de Cardiología Núcleo Guayas, a las autoridades de la Facultad de Ciencias Médicas de la Universidad de Guayaquil, y a Fuad Huamán Garaicoa, Daniel Tettamanti Miranda, Yan C. Duarte Cáceres y Alejandro A. Flor Márquez por su colaboración en este manuscrito. 


\section{Financiamiento}

Esta investigación no ha recibido ninguna beca específica de agencias de los sectores público, comercial, o sin ánimo de lucro.

\section{Conflicto de intereses}

Ninguno.

\section{Responsabilidades éticas}

Protección de personas y animales. Los autores declaran que los procedimientos seguidos se conformaron a las normas éticas del comité de experimentación humana responsable y de acuerdo con la Asociación Médica Mundial y la Declaración de Helsinki.

Confidencialidad de los datos. Los autores declaran que han seguido los protocolos de su centro de trabajo sobre la publicación de datos de pacientes.

Derecho a la privacidad y consentimiento informado. Los autores han obtenido el consentimiento informado de los pacientes y/o sujetos referidos en el artículo. Este documento obra en poder del autor de correspondencia.

\section{Bibliografía}

1. American Psychiatric Association, American Psychiatric Association, editors. Diagnostic and statistical manual of mental disorders: DSM-5. $5^{\text {th }}$ ed. Washington, D.C: American Psychiatric Association, 2013.

2. Prince M, Wimo A, Guerchet M, Ali G-C, Wu Y-T, Prina M. World Alzheimer Report 2015. The Global Impact of Dementia. An analysis of prevalence, incidence, cost and trends. 2015

3. Freire WB, Rojas E, Pazmiño L, Fornasini M, Tito S, Buendía P, et al. Encuesta Nacional De Salud, Bienestar y Envejecimiento: SABE I Ecuador 2009-2010. MIES

4. Petersen RC, Caracciolo B, Brayne C, Gauthier S, Jelic V, Fratiglioni L. Mild cognitive impairment: a concept in evolution. Journal of Internal Medicine. 2014;275(3):214-28.

5. Goldstein F, Levey A, Steenland N. High blood pressure and cognitive decline in mild cognitive impairment. Journal of the American Geriatrics Society. 2013;61(1):67-73.
6. Rocca WA, Petersen RC, Knopman DS, Hebert LE, Evans DA, Hall KS et al. Trends in the incidence and prevalence of Alzheimer's disease, dementia, and cognitive impairment in the United States. Alzheimer's \& Dementia. 2011;7(1):80-93

7. Whitney KA, Mossbarger B, Herman SM, Ibarra SL. Is the Montreal Cognitive Assessment superior to the Mini-Mental State Examination in detecting subtle cognitive impairment among middle-aged outpatient U.S. Military Veterans? Archives of Clinical Neuropsychology. 2012;27(7):742-8.

8. Knopman D, Petersen R. Mild cognitive impairment and mild dementia: a clinical perspective. Mayo Clinic Proceedings. 2014;89(10):1452-1459.

9. Petersen RC, Roberts RO, Knopman DS, Boeve BF, Geda YE, Ivnik RJ, et al. Mild cognitive impairment: ten years later. Arch Neurol. 2009;66(12):1447.

10. Brambati S, Belleville S, Kergoat M, Chayer C, Gauthier S, Joubert S. Single- and multiple-domain amnestic mild cognitive impairment: two sides of the same coin? 2018.

11. Csukly G, Sirály E, Fodor Z, Horváth A, Salacz P, Hidasi Z, et al. The differentiation of amnestic type $\mathrm{MCl}$ from the non-amnestic types by structural MRI. Frontiers in Aging Neuroscience. 2016;8.

12. Kwong LK, Neumann M, Sampathu DM, Lee VM, Trojanowski JQ. DP-43 proteinopathy: the neuropathology underlying major forms of sporadic and familial frontotemporal lobar degeneration and motor neuron disease. Acta Neuropathol. 2007;114(1):63. Epub 2007 May 10

13. Geda YE, Roberts, Knopman DS, Petersen RC, Christianson TJ, Pankratz VS, et al. Prevalence of neuropsychiatric symptoms in mild cognitive impairment and normal cognitive aging: population-based study. Arch Gen Psychiatry. 2008;65(10):1193.

14. Gabryelewicz T, Styczynska M, Pfeffer A, Wasiak B, Barczak A, Luczywek E, et al. Prevalence of major and minor depression in elderly persons with mild cognitive impairment--MADRS factor analysis. Int $J$ Geriatr Psychiatry. 2004;19(12):1168-72.

15. Langa $K$, Levine $\mathrm{D}$. The diagnosis and management of mild cognitive impairment. JAMA. 2014;312(23):2551.

16. Clarfield AM. The decreasing prevalence of reversible dementias: an updated meta-analysis. Arch Intern Med. 2003;163(18):2219-2229.

17. Cai X, Campbell N, Khan B, Callahan C, Boustani M. Long-term anticholinergic use and the aging brain. Alzheimer's \& dementia: The Journal of the Alzheimer's Association. 2013;9(4):377-385.

18. Karakaya T, Fußer F, Schroder J, Pantel J. Pharmacological treatment of mild cognitive impairment as a prodromal syndrome of Alzheimer's disease. Current Neuropharmacology. 2013;11(1):102-108.

19. In't Veld BA, Ruitenberg A, Hofman A, Stricker BH, Breteler MM. Antihypertensive drugs and incidence of dementia: the Rotterdam Study. Neurobiol Aging. 2001;22(3):407-12

20. Knopman D, Boland LL, Mosley T, Howard G, Liao D, Szklo M, et al. Cardiovascular risk factors and cognitive decline in middle-aged adults. Neurology. 2001;56(1):42.

21. Tzourio $C$, Dufouil $C$, Ducimetière $P$, Alpérovitch $A$. Cognitive decline in individuals with high blood pressure: a longitudinal study in the elderly. EVA Study Group. Epidemiology of Vascular Aging. Neurology. 1999;53(9):1948-52.

22. Muela HCS, Costa-Hong VA, Yassuda MS, Moraes NC, Memória CM Machado MF, et al. Hypertension severity is associated with impaired cognitive performance. J Am Heart Assoc. 2017;6(1).

23. Vicario A, Martínez CD, Baretto D, Díaz Casale A, Nicolosi L. Hypertension and cognitive decline: impact on executive function. J Clin Hypertens (Greenwich). 2005;7(10):598-604.

24. Yaffe K, Vittinghoff E, Pletcher MJ, Hoang TD, Launer LJ, Whitmer R, et al. Early adult to midlife cardiovascular risk factors and cognitive function. Circulation. 2014;129(15):1560-7.

25. Livingston G, Sommerlad A, Orgeta V, Costafreda SG, Huntley J, Ames D, et al. Dementia prevention, intervention, and care. Lancet. 2017;390(10113):2673. Epub 2017 Jul 20. 